\title{
FOURTEEN CASES OF FORCIBLE DILATATION OF THE OS BY MEANS OF THE BOSSI DILATOR.
}

\author{
By ERIC D. GAIRDNER, M.B. \\ House Surgeon, Glasgow Maternity Hospital.
}

THe following table shows a series of fourteen cases occurring during the last eight months in the practice of the Glasgow Maternity Hospital, in which artificial dilatation of the os was obtained by the use of either the Bossi or the Bossi-Frommer dilator. The cases have occurred in the ordinary indoor and outdoor practice of the Hospital and in the hands of various members of the medical staff.

Cases 1 and 2 were cases of eclampsia in which the fits were very severe; medicinal treatment had been of no effect.

Case 3 was a kidney case in which there was almost total suppression of urine. Diuretic treatment, either before or after the emptying of the uterus, was ineffectual; the skin and bowels were equally sluggish and resistant to remedies. The patient died five days after the operation in a comatose condition.

Case 4. This was another eclamptic case. There was a history of more than one fit before the patient was admitted. She was in labour when admitted, but a very severe fit occurring after admission it was deemed advisable to deliver without delay.

Case 5 was one of acute hydramnios. The patient was 4 months pregnant, and the uterus had distended from the normal size for that period of pregnancy to more than the size of a uterus at full term. Her general condition was such that it was determined to empty the uterus. Some hours before the operation was to have been done labour set in, and with such a degree of hæmorrhage as to render the immediate evacuation of the womb imperative.

In Case 6 the patient had been 36 hours in labour when admitted, and had been sent in from the country, a distance of 9 miles, in an ambulance waggon. The membranes had ruptured 12 hours previously. There was a considerable pelvic deformity, and the case, had it been seen earlier, would have been a suitable one for symphysiotomy, but under the circumstances such an operation was thought unadrisable. As she was much exhausted dilatation was effected, and the child with great difficulty delivered with forceps. The child's heart was very faintly beating at birth, but stopped before any attempt had been made to breathe. 
Forcible Dilatation of the Os

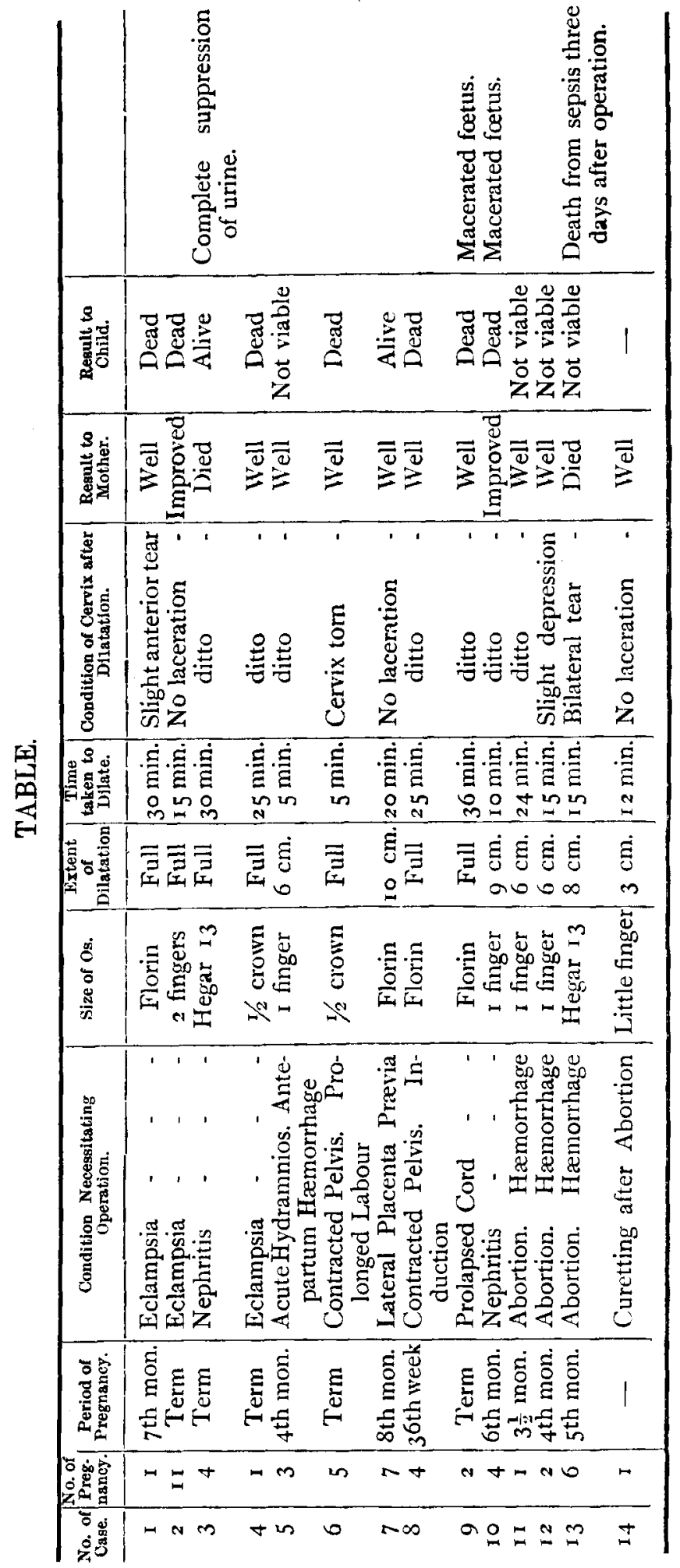


Case $i$ was one of lateral placenta prævia, the placenta overhanging the os. The ehild being alive and the patient in labour, it was determined to dilate as quickly as possible and deliver. This was effected without a great loss of blood and delivery of a live child obtained.

Case 8 was a case in which labour was induced on account of there being a considerable general contraction of the pelvis. A bougie was first introduced into the uterus and 24 hours later the dilatation was completed with the dilator. The child was only delivered with forceps with great difficulty, and was born dead.

In Case 9 the membranes had ruptured and the cord prolapsed 24 hours before the patient was seen. The pains which had been fairly strong had entirely ceased since the rupturing of the membranes. The cord, which was hanging from the vagina, was flaccid and black, and the fœetus was clearly felt to be macerated. It was therefore feared that sepsis would be set up unless the uterus was emptied without delay.

Case 10. This was another case of pregnancy complicated by kidney disease. There was anasarca and blindness. The quantity, however, of the urine, which was heavily loaded with albumen and casts, was not deficient and the skin and bowels acted freely. Nevertheless the patient's general condition became worse and worse, and when it was determined to empty the uterus she was all but comatose. T'en days after the operation she had slightly improved and was sent to the Royal Infirmary.

Cases 11 and 12 were cases of inevitable abortion in which there had been some considerable hæmorrhage.

In Case 13 the patient had had three floodings of grave severity, the last occurring 48 hours before admission. Since the last flooding there had been continuous oozing of blood. Before being admitted she had been seen outside by a doctor who had packed the vagina and sent her to hospital. The packing, which was soaked with discharge, was removed on the admission of the patient; it had a very offensive fotid smell. The oozing of blood still went on and the patient's condition became serious. It was then determined to empty the uterus. The patient was in a very critical condition for 24 hours after the operation, but she had evidently rallied from the operation and loss of blood, and seemed to be picking up when symptoms of septic infection set in and she died of sepsis, 3 days after the operation.

In Case 14 the uterus was curetted and the Bossi instrument used to dilate the os instead of Hegar's dilator. 
Cases 10,11, 12 and 14 occurred and were treated in the outdoor department of the hospital, the other cases of the series were treated indoors. The instrument used in the first three cases was Bossi's four-pronged dilator (Fig. 2). In all the other cases of the series the Bossi-Frommer eight-pronged instrument (Fig. 1) was used. With the latter instrument very much less force is necessary than with the former; the resistance of dilatation in full-time cases when the eight-pronged instrument is used being almost inappreciable.

When fully closed the eight-pronged instrument is equivalent in guage to a No. 13 Hegar dilator. The four-pronged instrument

Fig. 1.

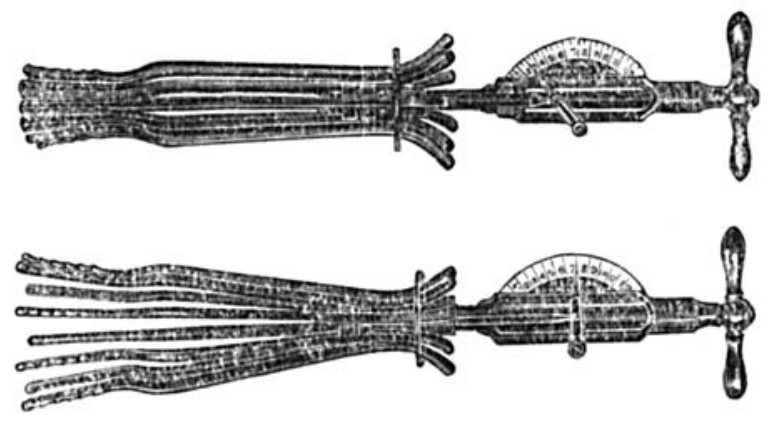

Fig. 2.
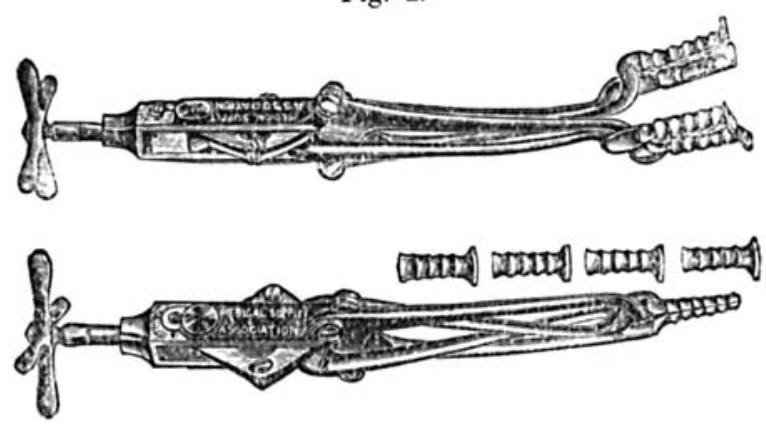

with caps off closes down to about a No. 11 Hegar; with the caps on the guage of the distal end is about equivalent to a No. 26 Hegar.

The most important consideration in regard to the use of the instrument is the condition of the os after dilatation has been accomplished. In all the cases of the series except cases 11-14 inclusive, the cervix had already been taken up before artificial dilatation was commenced. In Nos. 1 and 7 alone of this division of the series was the cervix in any way torn. In Case I there was a very slight laceration anteriorly, so slight as not to necessitate suturing. In 
Case 8 there was a somewhat ragged transverse tear which was sutured. It will be noticed that in this case full dilatation was accomplished in the short time of five minutes. When the cervix has been taken up and dilatation accomplished slowly, three minutes to a centimetre, it has been found that the operation is completed with little or no risk to the cervix.

In the remaining cases of the series, Nos. 11, 12, 13 and 14, the operation was performed in the early months of pregnancy, and before the cervix was taken up. In Case 13 there was an extensive bilateral tear after dilatation had been effected. The cervix in this case was fairly long and was also somewhat lengthened by being pulled upon by the volsellum forceps, and when the instrument was introduced into the cervix the closed prongs were not sufficiently long to reach through the internal os; consequently when dilatation was begun the prongs ripped right through the upper part of the cervical tissue. This accident demonstrates that there is a risk in obtaining dilatation when the cervix is not taken up owing to the prongs possibly being too short to reach through the internal os.

In Case 12, two days after delivery, some hæmorrhage occurred while an intra-uterine douche was being given; examination showed that a clot had been washed from a small pit or depression within the cervix which had evidently been caused by the tip of one of the prongs of the instrument indenting the cervical tissue. The hæmorrhage was easily controlled.

In Cases 11, 12 and 14 dilatation was effected without the administration of a general anæsthetic, the patients stating that the operation caused them no pain.

The above series of cases shows that with the Bossi-Frommer dilator forcible dilatation of the os can be rapidly and easily performed, and in cases where the cervix has been already taken up, with scarcely any risk of cervical laceration, provided that sufficient time be taken. Three minutes should be allowed to elapse during each centimetre of dilatation. Dilatation could probably be safely performed with greater speed than this, but such a rate has been found to give quite satisfactory results.

In cases where the cervix is not taken up there is greater risk of doing it damage owing, as already detailed, to the prongs not passing completely through the internal os. From reported cases I have not seen this danger referred to; it is the more liable to occur when the cervix is dragged upon and consequently lengthened by volsellum forceps being used to steady the organ. The dilator 
should therefore, if possible, be introduced without seizing the cervix with volsellum forceps, and when a slight degree of dilatation has been obtained the instrument should be closed and withdrawn, and an examination made before it is re-introduced, to ensure that the whole length of the cervix is being simultaneously dilated. Dilatation in these cases need not be any slower than in the former class, and can be accomplished with only a slightly greater degree of force.

Forcible dilatation of the os by this instrument is not accompanied by any greater degree of shock than is usual after any minor obstetric operation. In Case 13 the great amount of blood lost before the operation, added to the amount lost during the operation, was quite a sufficient cause to account for the patient's critical condition during the subsequent twenty-four hours, apart from any added shock due to the operation itself. The instrument, especially the eight-pronged variety, is therefore a very suitable and desirable one in cases where immediate evacuation of the uterus is considered necessary. 